\title{
Positron Emission Tomography with Deoxyglucose-F18 Imaging of Sleep
}

Monte S. Buchsbaum, M.D., Erin A. Hazlett, Ph.D., Joseph Wu, M.D., and William E. Bunney, Jr., M.D.

Positron emission tomography with deoxyglucose-F18 was obtained during nighttime sleep in 36 normal volunteers, 12 studied in rapid eye movement sleep (REM period 2), 12 in nonREM sleep, and 12 while awake with eyes closed. Metabolic rate was higher throughout the cortex in REM than nonREM sleep, with differences most marked in the cingulate and frontal cortex, thalamus, and visual association areas. Whole-brain metabolic rates in the waking condition were intermediate between those in REM and nonREM sleep. Metabolism in the primary visual cortex and parts of the lateral temporal lobe was relatively constant in the REM/nonREM conditions. REM sleep did not differ from either the nonREM or waking conditions in hemispheric lateralization of metabolic activity. Compared with REM sleep, nonREM sleep was associated with significantly lower metabolic rates in the temporal and occipital regions, as well as the thalamus.

[Neuropsychopharmacology 25:S50-S56, 2001]

(C) 2001 American College of Neuropsychopharmacology. Published by Elsevier Science Inc.
KEY WORDS: Sleep; Positron emission tomography (PET); Rapid eye movement sleep; Functional brain imaging; Glucose metabolism

Because sleep has traditionally been considered to be a period of "rest" (Adam and Oswald 1977; Benington and Heller 1995) rather than a process involving the specific activity of a single brain system, early sleep studies examined whole brain activity, measured blood hormone levels, and used a single representative cortical EEG lead. Attempting to characterize regional patterns, our group was the first to apply topographic EEG techniques to the study of sleep (Buchsbaum et al. 1982). Because EEG mapping approaches were limited to the assessment of surface electrical activity, our attention was redirected toward brain-imaging techniques, such as positron emission tomography (PET),

From the Neuroscience PET Laboratory, Department of Psychiatry, Mount Sinai School of Medicine, New York, NY, USA (MSB, $\mathrm{EAH}$ ) and the Brain Imaging Laboratory, University of California, Irvine, California, USA (JW, WEB).

Address correspondence to: Monte S. Buchsbaum, M.D., Department of Psychiatry, Box 1505, New York, NY, 10029-6574, Tel.: (212) 241-5287, Fax: (212) 423-0819, E-mail: monte.buchsbaum@mssm.edu that could explore activity in deeper brain structures. Our interest was also stimulated by the first quantitative studies of cerebral metabolism with deoxyglucose in monkeys during nonREM sleep (Kennedy et al. 1982; Nakamura et al. 1983). Human studies with PET and deoxyglucose-F18 (FDG) seemed the logical next step. In providing temporal and spatial resolution, PET had the potential to reveal important local brain mechanisms in the regulation of human sleep and wakefulness.

In this report, we review recent imaging studies and present a re-analysis of the earliest and still unique FDG-PET studies of nighttime, polygraphically staged sleep carried out by our group. The imaging program, which began in the late 1980s, was a collaboration between the Brain Imaging Center, University of California Irvine, headed by Dr. Monte S. Buchsbaum, and the Mental Health Clinical Research Center, University of California San Diego, headed by Dr. J. Christian Gillin. Projects conducted as part of that synergistic collaboration ranged from studies of metabolic activity during nighttime sleep in normal volunteers (Buchsbaum et al. 1989) and depressive patients (Ho et al. 1996); studies of the effects of sleep deprivation on waking brain metabolism in normal volunteers (Wu et al. 1991) and depres- 
sive patients (Wu et al. 1992, 1999); and the relationship of brain metabolism to dream content (Gottschalk et al. 1991), eye movements during dreaming sleep and wakefulness (Hong et al. 1995), and effects of sleeping medication (Gillin et al. 1996).

\section{BRIEF REVIEW: IMAGING STUDIES OF SLEEP}

Most studies indicate that rates of global cerebral metabolism rate and cerebral blood flow (CBF) are similar during REM sleep and wakefulness (Buchsbaum et al. 1989; Braun et al. 1998; Franzini 1992; Kuboyama et al. 1997; Lydic et al. 1991; Madsen et al. 1991; Madsen 1993; Maquet et al. 1996). Nevertheless, the specific patterns of metabolic activity throughout the brain show distinctive regional differences between REM and waking conditions. For example, limbic structures, including the cingulate gyrus, may be more metabolically active during REM sleep than during wakefulness, whereas higher order association areas, such as the orbital and dorsolateral regions of the frontal cortex and the inferior parietal cortex, may be relatively deactivated (Braun et al. 1998; Buchsbaum et al. 1989; Maquet et al. 1996; Nofzinger et al. 1997).

In comparisons of patterns of regional CBF in sleepdeprived normal subjects during wakefulness and different sleep stages (Andersson et al. 1998; Hofle et al. 1997; Kajimura et al. 1999; Maquet et al. 1996), $\mathrm{H}_{2}{ }^{15} \mathrm{O}$ uptake was found to be increased in limbic structures, including anterior cingulate, during REM sleep compared with wakefulness or nonREM sleep. Some studies (e.g. Braun et al. 1998) suggested that REM sleep was characterized by generalized activation of the brain (particularly pons, midbrain, caudate, paralimbic-limbic structures) without participation of regions in the frontoparietal areas that are normally involved in higher order analysis and integration of neural information. Maquet et al. (1996) particularly emphasized the activation of the amygdala during REM sleep. With its interactions with the anterior cingulate and posterior cortices, the amygdala might reactivate affective components of memories, bringing about the consolidation of memory traces during REM sleep. Similarly, Nofzinger et al. (1997, 2000), emphasizing the widespread activation of midline limbic and paralimbic structures, and temporal and insular cortex, in REM sleep compared with pre-sleep wakefulness, suggested that one function of REM sleep is the integration of neocortical activity with hypothalamic-basal forebrain regulatory and motivational mechanisms.

In a discussion of their $\mathrm{H}_{2}{ }^{15} \mathrm{O}$ CBF study in normal subjects, Braun et al. (1997) noted that centrencephalic regions, including brainstem, thalamus, and basal forebrain, are deactivated during slow wave sleep compared with pre-sleep wakefulness and reactivated during REM sleep. Similar changes were observed in anterior insula, anterior cingulate, and mesial temporal cortex. They suggested that the psychological characteristics of dreaming are accounted for by the relative deactivation of prefrontal cortex (which normally maintains "reality testing" during the waking state), the activated limbic system (which is responsible for the emotionality of dreams), and the activation of the hippocampal and parahippocampal system (which may recall the memory elements on which dreams are built). Braun et al. (1998) also found that CBF increased during REM sleep in extrastriate visual cortices but not in primary striate visual cortex. The observation of Braun et al. suggests that the visual experience of dreaming is not necessarily associated with activation of primary visual sensory areas. Moreover, they found that activity in extrastriate cortex correlated positively with that in limbic and paralimbic regions and negatively with that in lateral orbital and dorsolateral prefrontal cortices.

In a follow-up to our PET study comparing REM sleep and waking in normal volunteers, we investigated the neural substrates for rapid eye movements, specifically by correlating glucose metabolic rates in oculomotor areas with number of rapid eye movements during a 30-min period during wakefulness and REM sleep (Hong et al. 1995). Significant positive correlations emerged between number of eye movements during REM sleep and glucose metabolic rates in neuroanatomical areas corresponding to (a) the saccadic eye movement system (frontal eye field and the right dorsolateral prefrontal cortex), (b) midline attentional system (cingulate and medial frontal cortex, precuneus), and (c) parietal visual spatial attentional system (bilateral superior parietal lobules, right inferior parietal lobule). In general, similar correlations were found during wakefulness, with some exceptions. Thus, some of the same neural mechanisms may be involved in eye movements during REM sleep and wakefulness, and the cortex may play an important role in initiating eye movements in REM sleep, just as it does in wakefulness. Since some but not all studies suggest that eye movements "scan" the subjective dream during REM sleep, these results may further our understanding of the neurobiology of dreaming (Hobson 1988).

\section{RE-ANALYSIS OF UCI-UCSD STUDIES}

In the current re-analysis of our original quantitative image set (Buchsbaum et al. 1989), we carried out significance probability mapping (SPM) to complement our earlier region-of-interest (ROI) approach and for comparison with later studies that used SPM.

\section{Subjects and Procedures}

Thirty-six normal men (mean age $=26$ years, $\mathrm{SD}=7.0$ ) were studied during nonREM sleep $(n=12)$, REM sleep 
$(\mathrm{n}=12)$ and waking $(\mathrm{n}=12)$. Subjects maintained their normal sleep-wake patterns, retiring at 11 P.M. They were adapted to sleeping alone in a dark, quiet room during polygraphic recording of EEG, EOG, submental EMG and EKG, and with an i.v. catheter for one night before the study night. Polygraphic recordings were obtained and visually scored by standard criteria (Rechtschaffen and Kales 1971). The 36 subjects were obtained from a larger group of 48 subjects (eight subjects eliminated because of long sleep latency, poor sleep maintenance or dropping out after the accommodation night; four failed to meet the criterion of $75 \%$ or more of the first $32 \mathrm{~min}$ after FDG injection being in the appropriate sleep stage). No subject was scanned twice. For nonREM sleep, 11 subjects were studied during nonREM period \#1, one during nonREM period \#3; for REM sleep, subjects were in REM sleep an average of $88.3 \%$.

Before PET scanning, an individually molded, thermostatic plastic head holder was made for each subject to minimize head movement. Intravenous (i.v.) lines of $0.9 \%$ saline drip were inserted into both arms; a 3-m polyethylene tube extended out of the room so that FDG could be administered and blood withdrawn without disturbing the subject. One arm, wrapped in a hot pack for arterialization of venous blood, was used to withdraw blood samples $(2 \mathrm{ml})$ for determination of the kinetics of FDG; the other arm was used for i.v. administration of FDG. Polygraphic tracings of EEG, EOG, and EMG were continuously monitored by visual observation. About 5-10 min after the onset of nonREM sleep, REM period \#2, or a waking eyes-closed period, FDG (4-5 mCi) was administered i.v. over $30 \mathrm{~s}$ in one arm while blood samples were withdrawn from the other arm. After 32 min of FDG uptake, the subject was awakened and transferred to the adjacent scanning room. Nine planes at $10-\mathrm{mm}$ increments and parallel to the canthomeatal line were obtained 45-100 min after FDG injection. Full details of scanning procedures are provided in our initial report (Buchsbaum et al. 1989).

Scans were transformed to glucose metabolic rate, expressed as absolute cerebral glucose metabolic rate in $\mathrm{mmol} / 100 \mathrm{~g} / \mathrm{min}$ (Buchsbaum et al. 1989). Each image had an edge outline drawn with an automated algorithm and had nine midline points identified according to our standard method (Buchsbaum et al. 1998). The images were normalized in shape to the average outline for the group for significance probability mapping (SPM) (Bartels and Subach 1976). The image was adjusted to contain the same number of rows as the average image, and the average row length for each row was computed from the midline to the edge pixel; the final image was adjusted so each row was symmetrical with a length equal to the average of the left and right sides. This allowed statistical mapping of asymmetry since each row had equal numbers of pixels to the left and right of the midline. Comparisons of metabolic rate were carried out using SPM with unpaired $t$-tests comparing each voxel.

\section{RESULTS}

\section{REM vs. NonREM Sleep}

The cortex had higher metabolism in REM than nonREM sleep (Figure 1), with intermediate rates during waking. Differences were widespread and shown by nearly all subjects. Higher metabolic rates during REM than nonREM sleep were confirmed by SPM, with differences being most marked in midline frontal, cingulate, and dorsolateral prefrontal regions (Figure 2) and in the 3- to 6-micromole range. The thalamus also showed higher values in REM than nonREM sleep. Primary visual cortex seemed little affected, although higher values in visual association cortex during REM than nonREM sleep were observed.

\section{NonREM Sleep vs. Awake}

NonREM sleep was associated with significantly lower metabolic rates in temporal and occipital regions and in the thalamus, but not the anterior temporal, insula or some regions of the frontal lobe (Figure 3). In assessments of possible asymmetrical patterns, difference scores for each pixel (left minus right) and $t$-tests comparing the difference scores revealed a tendency for higher right hemisphere values, but few findings of asymmetry achieved $p<.05,2$-tailed (Figure 4) and none survived more stringent statistical resampling to control for multiple comparisons. No asymmetry pixel clusters were significant for REM vs. nonREM sleep.

\section{DISCUSSION}

These data indicate a global metabolic decrease in nonREM sleep and higher activity throughout the cingulate, frontal lobe and thalamus in REM sleep compared with both nonREM sleep and wakefulness. As the data are in absolute units, the global changes can be appreciated in a way unavailable when only relative flow levels or metabolic rates are analyzed. Thus, for example, if every pixel is referenced to the global brain metabolic rate, increases in the cingulate in REM sleep could reflect small global cortical decreases with the cingulate unchanged. The pervasive increase in metabolic activity in the cingulate during REM sleep in our original study (Buchsbaum et al. 1989) is more extensively portrayed in the current analysis with SPM, extending from the orbital region through the dorsal arch of the cingulate. Our findings of change in absolute rates of glucose utilization provide important confirmatory information for the relative $\mathrm{H}_{2}{ }^{15} \mathrm{O}$ data that $\mathrm{CBF}$ in the 


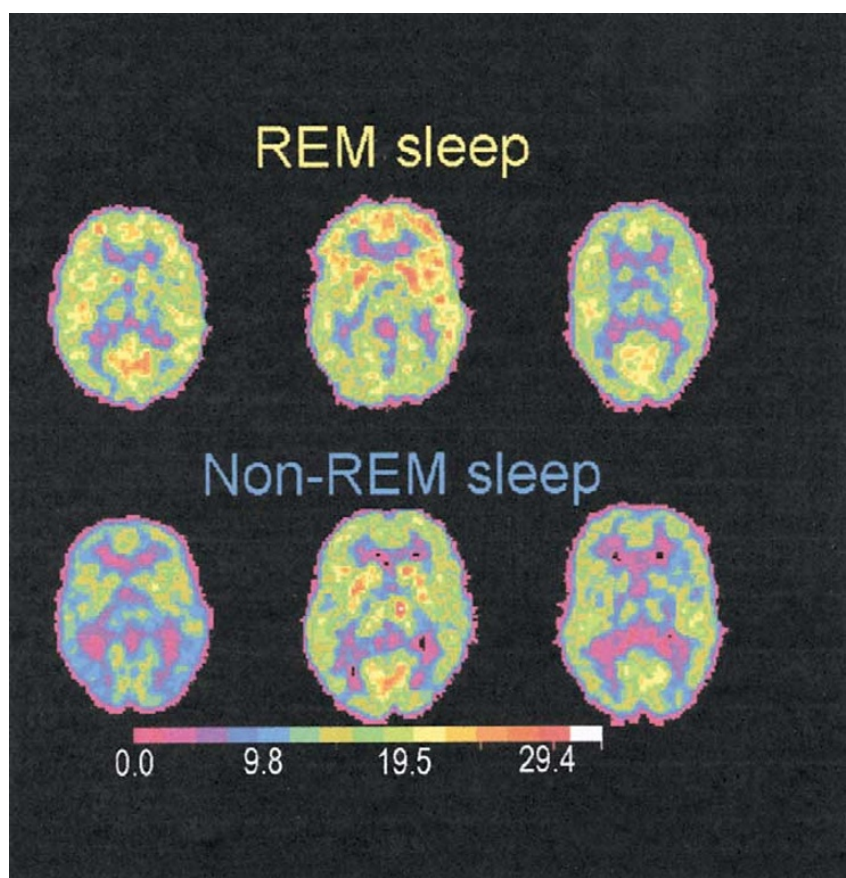

Figure 1. FDG uptake in three typical healthy individuals during REM sleep (top) and three typical individuals in nonREM sleep (bottom). Images are presented on same color scale as shown in color bar in micromoles glucose $/ 100 \mathrm{~g} / \mathrm{min}$. Note higher metabolic rates in REM sleep, especially in the cingulate.

Figure 2. SPM maps of metabolic rate during REM and nonREM sleep. Top: average metabolic rate images for REM and nonREM (left scale bar) and pixel-by-pixel differences in metabolic rate (right scale bar). Bottom: SPM maps with $t$-test threshold at $p<.05$ and $p<.005$.
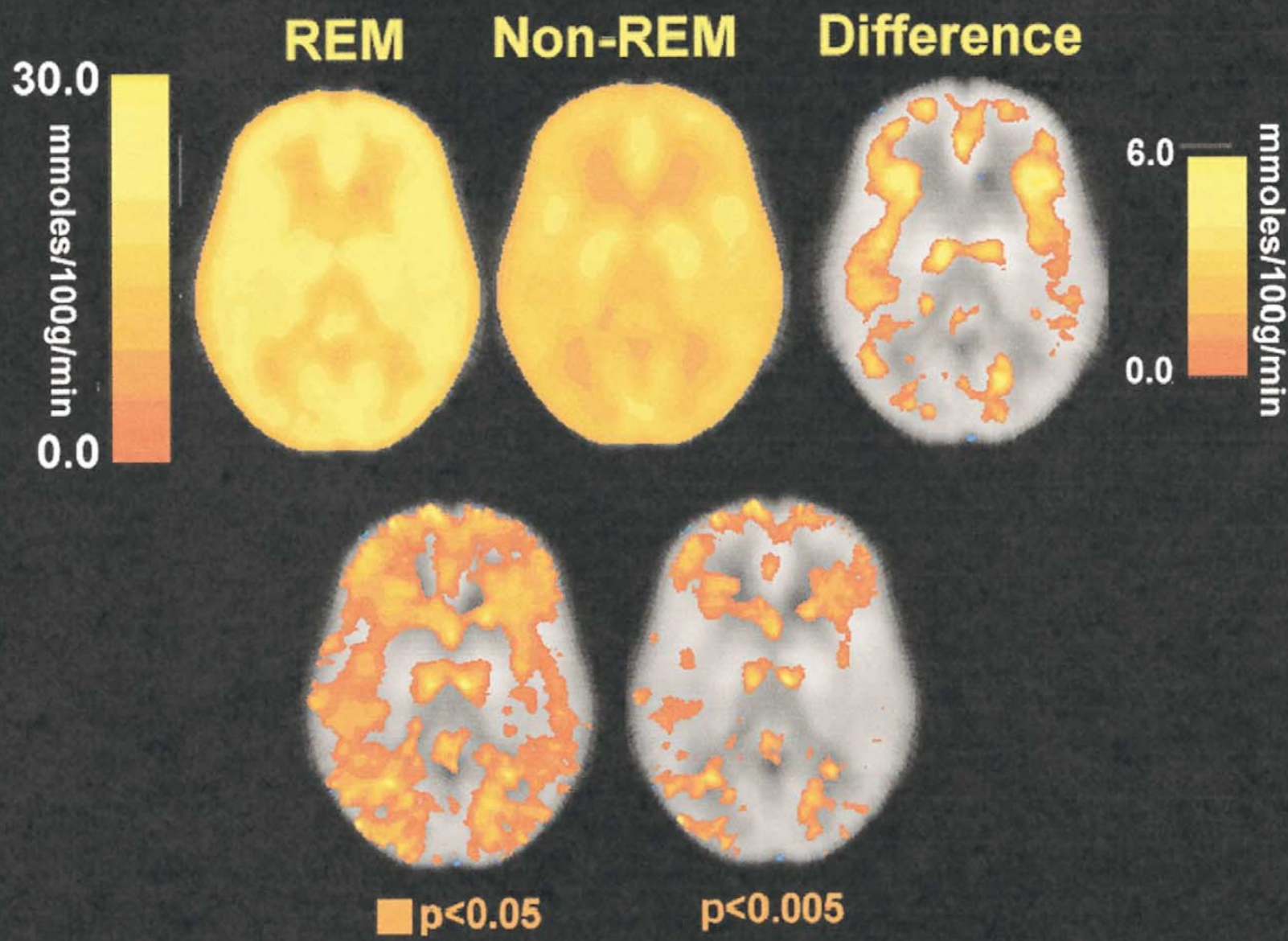




Figure 3. REM and nonREM sleep. Top: T-test comparison of six levels of the brain with axial level indicated in Talairach coordinates $(p<.05,2$-tailed). The cingulate from the orbital portion (BA 25) to the more dorsal portion (BA 24), thalamus and entire frontal cortex are prominently higher in REM than nonREM sleep. Bottom: T-test comparisons ( $p<.005,2$-tailed). Cingulate, ventral thalamus, and frontal lobe show prominent effects.

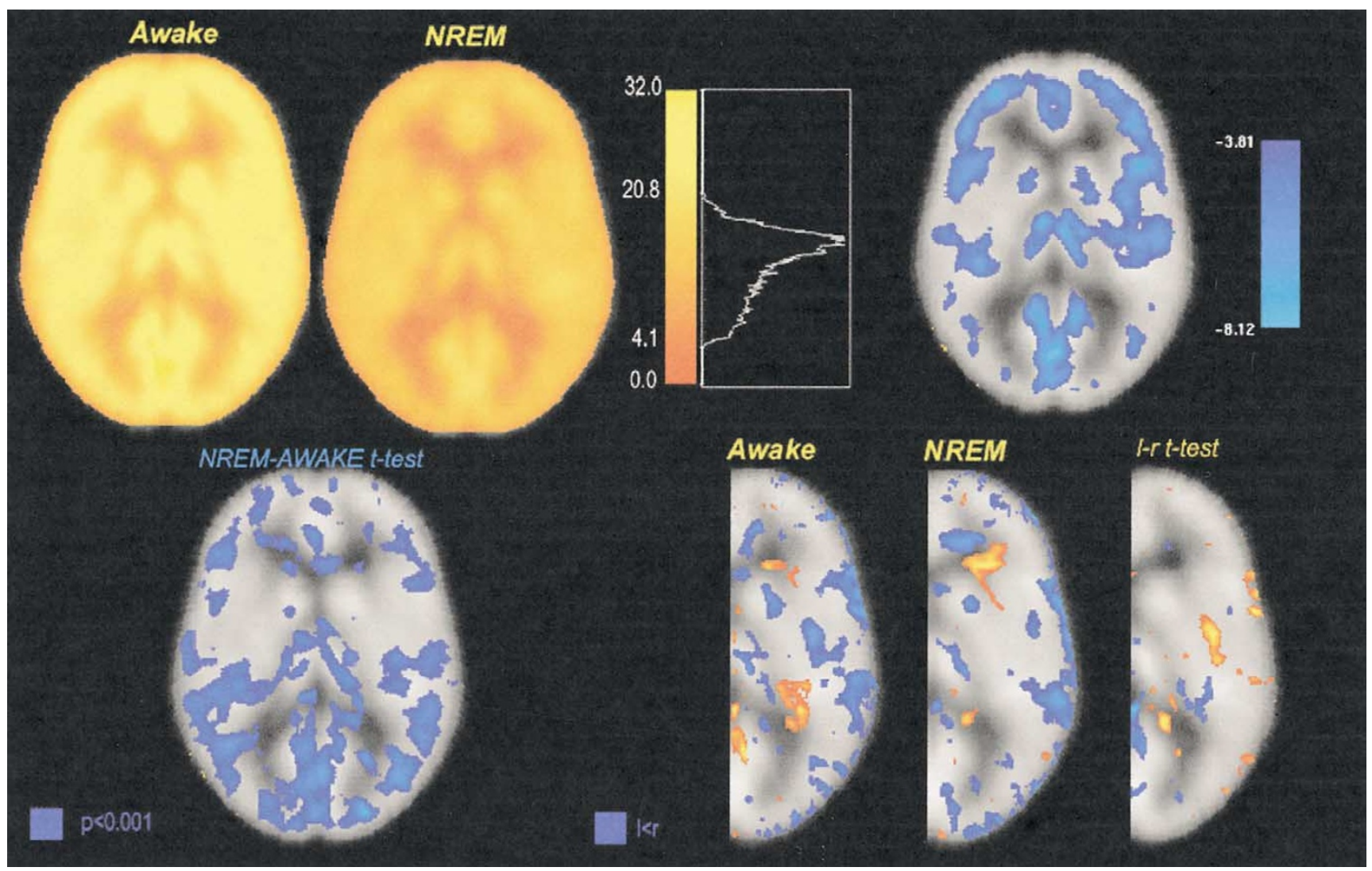

Figure 4. Mean FDG-PET for 12 healthy subjects awake and 12 subjects in nonREM (NREM) sleep. Top from left to right: Mean PET $(z=+12)$ for awake and for nonREM, frequency histogram of all pixels in nonREM sleep with peak at 18 micromoles/ $100 \mathrm{~g} / \mathrm{min}$, mean difference displayed as nonREM minus awake with color bar for metabolic rate difference in micromoles of glucose $/ 100 \mathrm{~g} / \mathrm{min}$. Bottom row from left to right: $t$-test comparison map for nonREM vs. awake, and three left minus right $t$-test asymmetry maps for awake, nonREM, and left minus right $t$-test for difference scores between nonREM and awake. For asymmetry maps, blue indicates left hemisphere lower than right, $p<.05$ and for left minus right $t$-test for difference scores, colored areas have significantly different asymmetry, in awake and nonREM, $p<.05$, 2-tailed. 
cingulate gyrus is increased in REM sleep in REMdeprived subjects (Braun et al., 1998) and the findings for the cingulate region in sleep-deprived subjects reported by Maquet et al. (1996). The findings of Braun and Maquet and their associates might also have partly reflected arousal features of an unfamiliar sleep environment in which subjects were of necessity immobilized in the PET scanner or the effects of changes in normal sleep rhythm. Our results suggest that the REMassociated increase in the cingulate does not depend on these experimental maneuvers but also appears in normal subjects sleeping in beds at night after a night of laboratory accommodation.

PET has been used for more than a decade to study patterns of brain activity during nighttime sleep. Some investigators have favored $\mathrm{H}_{2}{ }^{15} \mathrm{O}$ methods, while others have used FDG. Some have reported absolute measures, while others have used relative measures. Statistical analytic approaches have ranged from hypothesisdriven ROI methods to exploratory SPM. Each method has its strengths and limitations. The FDG-PET method permits a more "naturalistic" setting: that is, subjects sleep in beds (not in the PET scanner), and the data generated when awakened subjects are later moved to the PET scanner reflect the subject's brain activity while asleep in bed, not while awake in the scanner. Polysomnographic measures are also somewhat more easily obtained in the standard sleep-laboratory setting permitted by the FDG method. Both absolute glucose quantification and relative measures of metabolism may be routinely obtained with the FDG method, but absolute measures are more problematic with the CBF approach. Another point of contrast between the two tracers is the time interval required for uptake: approximately 30 min for FDG compared with an interval of seconds for the $\mathrm{H}_{2}{ }^{15} \mathrm{O}$ blood flow technique. The spatial resolution of FDG-PET is not limited by the reduced counting time and the 3 - to 4 -fold longer $8-\mathrm{mm}$ path of the positron emitted by O-15. Indeed, the $4.5-\mathrm{mm}$ or better resolution available with modern scanners with FDG as tracer is diminished substantially with $\mathrm{H}_{2}{ }^{15} \mathrm{O}$. Some brain regions involved in sleep regulation are too small, however, to be accurately assessed with either FDG or $\mathrm{H}_{2}{ }^{15} \mathrm{O}$, and their study will require technical advances in scanner instrumentation. In other relevant brain regions that are small but within the theoretical resolution of modern scanners, confidence in the accuracy of results obtained with FDG is greater than with $\mathrm{H}_{2}{ }^{15} \mathrm{O}$. Although FDG has an advantage in spatial resolution, its temporal resolution is inferior to that of $\mathrm{H}_{2}{ }^{15} \mathrm{O}$. If polysomnographic measures are used in combination with $\mathrm{H}_{2}{ }^{15} \mathrm{O}$ PET, then CBF during REM sleep is unlikely to be contaminated by activity from adjacent nonREM sleep. With the longer uptake period of FDG, it is necessary to compromise by defining the "REM period" as metabolic activity in which $75 \%$ or more of the first 32 min after FDG injection was in the appropriate sleep stage. A related difficulty of the FDG method is that data obtained from a subject who switches out of REM sleep after only 5-10 min of uptake must be discarded. For logistic reasons, therefore, several subjects must be studied during the same night to ensure that usable data will be obtained from at least one. The $\mathrm{H}_{2}{ }^{15} \mathrm{O}$ method, with its shorter uptake time, obviates this problem but has the disadvantage of requiring subjects who are able to sleep soundly while confined in a PET scanner, a barrier to studies of insomniacs and some psychiatric patients.

In view of the differing strengths and limitations of available PET methodologies, it is encouraging that findings obtained with different techniques have, in general, been in good agreement. With the development of higher resolution PET instruments and the use of other high-resolution techniques such as functional magnetic resonance, the next decade of sleep studies offers the promise of a more refined understanding of the brain's activity in human sleep.

\section{REFERENCES}

Adam K, Oswald I (1977): Sleep is for tissue restoration. J R Coll Physicians London 11:376-388

Andersson JLR, Onoe H, Hetta J, Lindstrom K, Valind S, Lilja A, Sudin A, Fasth K-J, Westerberg G, Broman J-E, Watanabe Y, Langsrom B (1998): Brain networks affected by synchronized sleep visualized by positron emission tomography. J Cereb Blood Flow Metab 18: 701-715

Bartels P, Subach J (1976): Significance probability mappings and automated interpretation of complex pictorial scenes. In Preston E, Onoe M (eds), Digital Processing of Biomedical Imagery. New York, Academic Press, pp 101-114

Benington JH, Heller HC (1995): Restoration of brain energy metabolism as the function of sleep. Prog Neurobiol 45:347-360

Braun AR, Balkin TJ, Wesenten NJ, Carson RE, Varga M, Baldwin P, Selbie S, Belenky G, Herscovitch P (1997): Regional cerebral blood flow throughout the sleepwake cycle. Brain 120:1173-1197

Braun AR, Balkin TJ, Wesensten NJ, Carson RE, Varga M, Baldwin P, Belenky G, Herscovitch P (1998): Dissociated pattern of activity in visual cortices and their projections during human rapid eye movement sleep. Science 279:91-95

Buchsbaum MS, Gillin JC, Wu J, Hazlett E, Sicotte N, Dupont R, Bunney WE (1989): Regional cerebral glucose metabolic rate in human sleep assessed by positron emission tomography. Life Sci 45:1349-1356

Buchsbaum MS, Mendelson WB, Duncan WC, Coppola R, Kelsoe J, Gillin JC (1982): Topographic cortical mapping of EEG sleep stages during daytime naps in normal subjects. Sleep 5:248-255 
Buchsbaum MS, Tang CY, Peled S, Gudbjartsson H, Lu D, Hazlett EA, Downhill J, Haznedar M, Fallon JH, Atlas SW (1998): MRI white matter diffusion anisotropy and PET metabolic rate in schizophrenia. NeuroReport 9: 425-430

Franzini C (1992): Brain metabolism and blood flow during sleep. J Sleep Res 1:3-16

Gillin JC, Buchsbaum MS, Valladares-Neto DC, Hong CC-H, Hazlett E, Langer SZ, Wu J (1996): Effects of zolpidem on local cerebral glucose metabolism during non-REM sleep in normal volunteers. Neuropsychopharmacology 15:302-313

Gottschalk LA, Buchsbaum MS, Gillin JC, Wu JC, Reynolds CA, Herrera DB (1991): Anxiety levels in dreams. Brain Res 538:107-110

Ho AP, Gillin JC, Buchsbaum MS, Wu JC, Abel L, Bunney WE (1996): Brain glucose metabolism during non-rapid eye movement sleep in major depression. Arch Gen Psychiatry 53:645-652

Hobson JA (1988): The Dreaming Brain. New York, Basic Books

Hofle N, Paus T, Reutens D, Fiset P, Gotman J, Evans AC, Jones BE (1997): Regional cerebral blood flow changes as a function of delta and spindle activity during slow wave sleep in humans. J Neurosci 17:4800-4808

Hong CC, Gillin JC, Dow BM, Wu J, Buchsbaum MS (1995): Localized and lateralized cerebral glucose metabolism associated with eye movements during REM sleep and wakefulness. Sleep 18:570-580

Kajimura N, Uchiyama M, Takayama Y, Uchida S, Uema T, Kato M, Sekimoto M, Watanabe T, Nakajima T, Horikoshi S, Ogawa K, Nishikawa M, Hiroki M, Kudo Y, Matsuda H, Okawa M, Takahashi K (1999): Activity of midbrain reticular formation and neocortex during the progression of human non-rapid eye movement sleep. J Neurosci 19:10065-10073

Kennedy C, Gillin JC, Mendelson WB, Suda S, Miyaoka M, Ito M, Nakamura RK, Storch FI, Pettigrew K, Mishkin M, Sokoloff L (1982): Local cerebral glucose utilization in non-REM sleep. Nature 297:325-327

Kuboyama T, Hori A, Sato T, Mikami T, Yamaki T, Ueda S (1997): Changes in cerebral blood flow velocity in healthy young men during overnight sleep and while awake. Electroencephalogr Clin Neurophysiol 102:125-131
Lydic R, Baghdoyan H, Hibbard L, Bonyak E, DeJoseph M, Hawkins R (1991): Regional brain glucose metabolism is altered during rapid eye movement sleep in the cat. J Comp Neurol 304:517-529

Madsen PL (1993): Blood flow and oxygen uptake in the human brain during various states of sleep and wakefulness. Acta Neurol Scand 88(Suppl) 148:5-25

Madsen PL, Holm S, Vorstrup S, Friberg L, Lassen NA, Wildschiodtz G (1991): Human regional cerebral blood flow during rapid-eye-movement sleep. J Cereb Blood Flow Metab 11:502-507

Maquet P, Peters JM, Aerts J, Delfiore G, Dequeldres C, Luxen A, Franck G (1996): Functional neuroanatomy of human rapid eye movement sleep and dreaming. Nature 383:163-165

Nakamura RK, Kennedy C, Gillin JC, Suda S, Ito M, Storch F, Mendelson WB, Sokoloff L, Mishkin M (1983): Hypnogenic center theory of sleep. Brain Res 268:373-376

Nofzinger EA, Mintun MA, Wiseman MB, Kupfer DJ, Moore RY (1997): Forebrain activation in REM sleep. Brain Res 770:192-201

Nofzinger EA, Price JC, Meltzer CC, Buysse DJ, Villemagne VL, Miewald JM, Sembrat RC, Steppe DA, Kupfer DJ (2000): Towards a neurobiology of dysfunctional arousal in depression. Psychiatry Res 98:71-91

Rechtschaffen A, Kales A (1971): A manual of standard techniques and scoring system for sleep stages in human subjects. Washington, DC, US Government Printing Office

Wu JC, Gillin JC, Buchsbaum MS, Hershey T, Hazlett E, Sicotte N, Bunney WE (1991): The effect of sleep deprivation on cerebral glucose metabolic rate in normal humans assessed with positron emission tomography. Sleep 14:155-162

Wu JC, Gillin JC, Buchsbaum MS, Hershey T, Johnson JC, Bunney WE (1992): Effect of sleep deprivation on brain metabolism of depressed patients. Am J Psychiatry 149:538-543

Wu J, Buchsbaum MS, Gillin JC, Tang C, Cadwell S, Wiegand M, Najafi A, Klein E, Hazen K, Bunney WE (1999): Prediction of antidepressant effects of sleep deprivation by metabolic rates in the ventral anterior cingulate and medial prefrontal cortex. Am J Psychiatry 156:11491158 